\title{
About notation
}

Attempts have been made to keep a single notation for various quantities discussed throughout this book. In some cases, small variations were accepted for consistency with norms, market usage, and original reference works.

In general, in this text, bold capital letters denote matrices, lowercase bold letters denote vectors, and italicized letters denote scalar magnitudes.

The letter T overwritten to the right of an array indicates its transpose, that is, permutation of rows by columns. An exponent -1 to the right of a matrix indicates its inverse.

Two vertical bars to the right and left of a matrix or vector denote a norm of the same; in the case of a scalar, they denote an absolute value. 
